\title{
A case of tooth fracture occurred upon medicating bisphosphonate for an elderly person: Preservation therapy and responses for Stage 0 of bisphosphonate-related osteonecrosis of jaw
}

\author{
Noriko Suzuki ${ }^{1}$, Hitoshi Oguchi ${ }^{1}, Y_{\text {Y Yamauchi }}{ }^{1}$, Yasuyo Karube ${ }^{1}$, Yukimi Suzuki ${ }^{1}$, \\ Noriyasu Hosoya ${ }^{2}$
}

Correspondence: Dr. Hitoshi Oguchi

Email: oguchi-h@tsurumi-u.ac.jp
'Department of Geriatric Dentistry, School of Dental Medicine, Tsurumi University, Yokohama, Japan, 2Department of Endodontology, School of Dental Medicine, Tsurumi University, Yokohama, Japan

\section{ABSTRACT}

This case report aimed to report the progress of preservation therapy and response of symptoms and signs for Stage 0 of bisphosphonate-related osteonecrosis of jaw (BRONJ). A 68-year-old female was recognized having a tooth at the left upper first molar fracture upon medicating bisphosphonate (BP) in 2007. At that time, the extraction of the tooth was an absolute contraindication. Therefore, we performed preservation therapy. We observed the symptoms and signs every month. After 5 months, swelling and redness in the entire first molar tooth were seen and fistula formed partly. Bone exposure was not seen. We administrated antibiotics immediately. As a result, symptoms disappeared. On April 10,2009 , the patient visited us as she felt a sense of incongruity in the lower left first and second molar teeth. Clinically, there were no symptoms of pain. However, we observed the radiolucent finding in about $5 \mathrm{~mm}$ diameter at apical position by X-ray photography; we considered a possibility of Stage 0 for BRONJ. We immediately administered medicine for 5 days and the symptoms disappeared. At present, no inflammation with signs and symptoms at the upper left first molar and lower left first, second molar parts is shown. We performed preservation therapy for tooth fracture case medicating of BP. Immediate responses for inflammation and symptoms of the Stage 0 of BRONJ have led to success. Hence, dentists should perform regular clinical observation, and enough education to the patient for BRONJ is necessary.

Key words: Bisphosphonate-Related Osteonecrosis of Jaw, preservation therapy, Stage 0, tooth fracture

\section{INTRODUCTION}

Bisphosphonate (BP) is an extremely effective medicine for osteoporosis and mitigation of pain in bone metastasis of mammalian cancer. As a result, $\mathrm{BP}$ has been the position as the standard treatment medicine of osteoporosis and mammalian cancer.

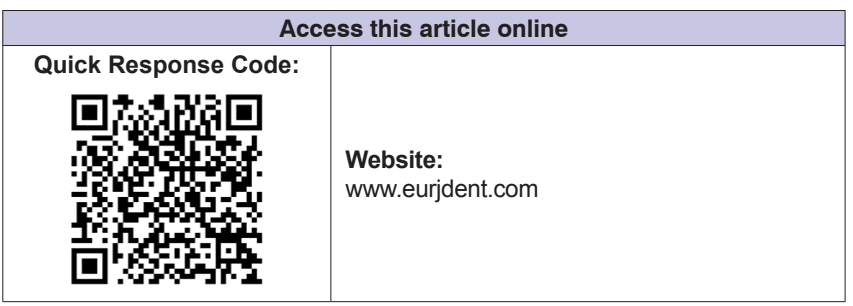

However, Wang et al., ${ }^{[1]}$ Marx, ${ }^{[2]}$ and Migliorati ${ }^{[3]}$ reported that medicating $\mathrm{BP}$ used for osteoporosis and metastasis of the bone for mamma cancer

This is an open access article distributed under the terms of the Creative Commons Attribution-NonCommercial-ShareAlike 3.0 License, which allows others to remix, tweak, and build upon the work non-commercially, as long as the author is credited and the new creations are licensed under the identical terms.

For reprints contact: reprints@medknow.com

How to cite this article: Suzuki N, Oguchi H, Yamauchi Y, Karube Y, Suzuki Y, Hosoya N. A case of tooth fracture occurred upon medicating bisphosphonate for an elderly person: Preservation therapy and responses for Stage 0 of bisphosphonate-related osteonecrosis of jaw. Eur J Dent 2017;11:258-63.

DOI: 10.4103/ejd.ejd_264_16 
caused Bisphosphonate-Related Osteonecrosis of Jaw (BRONJ) after operation of oral (including extraction and incision) or natural incidence in 2003. Till now, numerous researchers have reported similar cases of BRONJ. ${ }^{[4]}$ In Japan, Takahashi et al. reported that the first case of BRONJ was documented in 2006. ${ }^{[8]}$

From previous reports, the estimated cumulative incidence of this side effect for patients taking oral BP, values from $0.01 \%$ to $4 \%$, can be found depending on the source consulted. ${ }^{[9]}$ In an extensive examination conducted in Australia, the frequency of BRONJ is $0.05 \%-0.1 \%$ in whole of BP-medicated patients. ${ }^{[10]}$ Furthermore, Urade et al. ${ }^{[11]}$ and Yoneda et al. ${ }^{[12]}$ reported that the relative ratio of $\mathrm{BRONJ}$ related to oral $\mathrm{BP}$ was greater in Japan than in the United States and the European Union. The problems of definite mechanism are unknown having no conclusive clinical treatment method of BRONJ.

On the other hand, Mawardi et al. ${ }^{[13]}$ proposed the "Stage 0 " concept, a potential stage of BRONJ before development of a clinically defined BRONJ in 2009. As a result, the concept of the Stage 0 for BRONJ not accompanied with bone exposure has been proposed by the American Association of Oral and Maxillofacial Surgeons (AAOMS) and the symptoms and corresponding methods regarding Stage 0 have been described..$^{[14]}$

This time, we have experimented a tooth fracture case performed by preservation in BP intraoral administering. Furthermore, during the time course of observation, the patient complained discomfort at the lower left first and second molar teeth part later. From X-ray photograph, we considered a possibility of Stage 0 for BRONJ, therefore antibacterial medicine was administrated immediately, and then symptoms disappeared.

The purpose is to report the first successful case of tooth fracture as a result of preservation therapy and responses for Stage 0 for BRONJ through the tooth fracture case.

\section{CASE REPORT}

A 68-year-old female consulted for the first time on February 29, 2007. Her chief complaint was pain in the upper molar. On the diagnosis of osteoporosis in August 2006, intraoral medication of BP (25 mg of sodium risedronate hydrate [Actonel $\left.{ }^{\circledR}\right]$ ) was started.
Currently, the patient complained about volatile spontaneous pain in the upper left first molar tooth. Clinical findings (redness, swelling, etc.) were not seen, and X-ray photography was normal [Figure 1]. Since the pain disappeared after 1 week, we decided to observe progress. In April and May 2007, since slight swelling was seen, $300 \mathrm{mg}$ a day of cefdinir and $180 \mathrm{mg}$ a day of loxoprofen sodium hydrate for 5 days were administered. Symptoms alleviated and progress had to be observed again. However, in August, percussion pain was observed in the first molar tooth, and clear tooth fracture was seen at mesiodistal path. In dental photography, expansion of dental ligament cavity was not observed [Figure 1].

\section{Clinical diagnosis}

A diagnosis of tooth fracture of the upper left first molar tooth was made.

\section{Treatment and progress}

At first, extraction treatment was considered; however, the patient wished to preserve the tooth as long as possible. Furthermore, since the patient was under medication of BP, we consulted an oral and maxillofacial surgeon. Finally, we selected preservation therapy due to administrating BP. From the clinical and radiographic findings, a diagnosis of symptomatic chronic apical periodontitis was made, and root canal treatment was suggested to the patient. To prevent further tooth fracture, the entire crown part of the first molar tooth was wiring fixed with $0.25 \mathrm{~mm}$ diameter wire and later fixed with adhesive resin composite. Moreover, the opposite tooth was adjusted to prevent occlusion. A root canal access cavity was established followed by rubber dam isolation. Clinical examination revealed three root canal openings in each of the mesiobuccal, distobuccal, and palatal root after extirpating the necrotic pulp tissue. Furthermore, a crack was found in the floor of pulp chamber. It was sealed with flowable adhesive resin composite to prevent leakage. The root canals

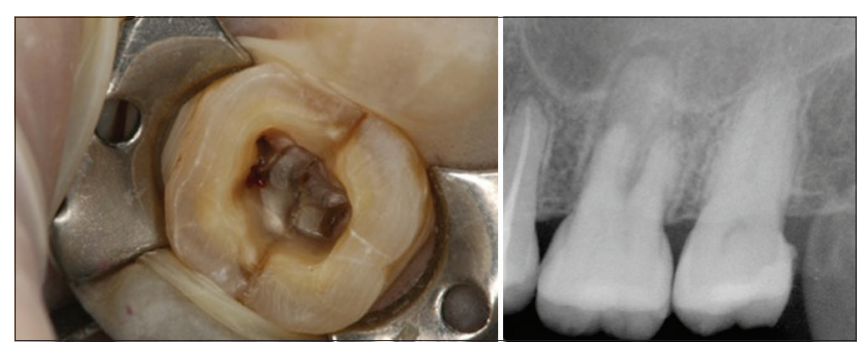

Figure 1: After pulpectomy and the root filling, the entire crown part of tooth was fixed wiring with $0.25 \mathrm{~mm}$ diameter wire and later fixed with adhesive resin composite 
were instrumented with the stainless steel files and copious irrigation with $3 \% \mathrm{NaOCl}$. The root canal was filled with gutta-percha points and root canal sealer by lateral condensation method. Since the patient was under BP medication, we informed the patient about the possibility of jaw bone necrosis reported earlier caused by BP and we observed progress at the rate of once a month. We offered the patient to visit us immediately, once clinical symptoms such as pain appear. Later, progress was good and the patient was steadily getting better with no pain. In January 2008, the patient complained about pain in the same area and therefore she was advised to visit us immediately. Swelling and redness in the entire first molar tooth were seen and abscess formed partly [Figure 2: left]. Spontaneous pain and percussion pain were observed. Bone exposure was not seen. Dental photography showed clear expansion of dental ligament cavity in each root [Figure 2: right]. Since administering cefdinir caused stomach discomfort previously, medication was changed to 5 days' administration of $200 \mathrm{mg}$ /day of clarithromycin, and the clinical symptoms disappeared. Moreover, again in November 2008, swelling was seen around the same area. There was no redness and pain. However, dental photography showed expansion of dental ligament cavity. Through similar prescription as provided earlier, symptoms disappeared [Figure 3]. On April 10, 2009, the patient visited us as she felt a sense of incongruity in the lower left first and second molar teeth. Clinically, there were no signs of pain, swelling, redness, and tooth fracture. However, we observed the radiolucent

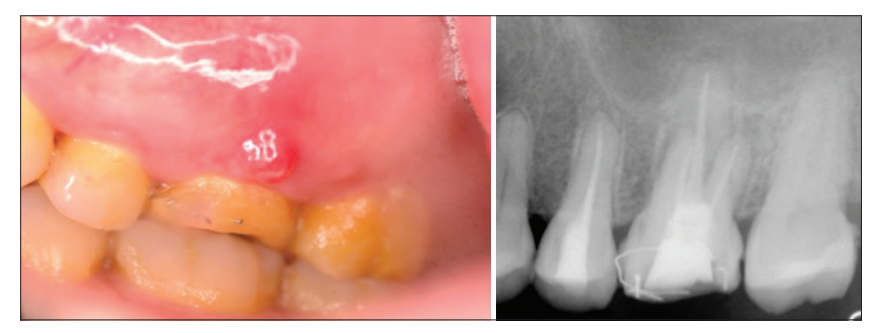

Figure 2: Swelling, redness, and abscess were seen clinically, and clear expansion of dental ligament cavity in each root as shown in X-ray photography. However, bone exposure was not seen

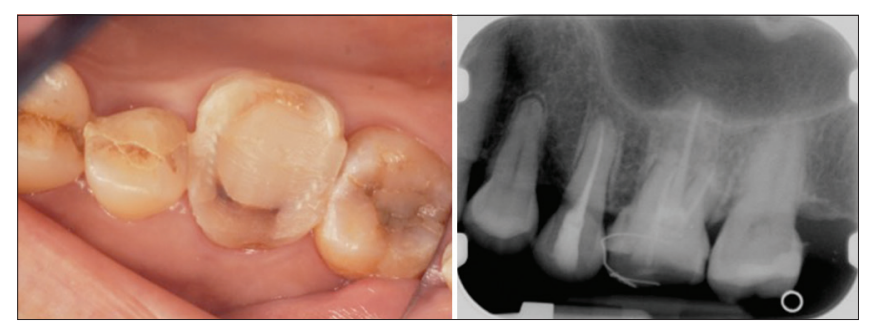

Figure 4: Radiolucent findings (upper left first photograph). We considered a possibility of Stage 0 for Bisphosphonate-Related Osteonecrosis of Jaw discussing with radiologist finding in about $5 \mathrm{~mm}$ diameter at apical position from the lower left first to second molar teeth by X-ray photography [Figure 4]. We considered a possibility of Stage 0 for BRONJ [Figure 5]. Therefore, we immediately administered medicine (clarithromycin) for 5 days and symptoms disappeared. On January 18, 2010, we have cemented the full metal crown. Later, we performed progress observation once a month and educated immediate consult of our department if symptoms or signs such as pain and swelling occur. At present, there is no inflammation with signs and symptoms at the upper left first molar and lower left first, second molar parts.

\section{DISCUSSION}

BRONJ is defined to have three characteristics as follows: (1) current or previous treatment with a $\mathrm{BP},(2)$ exposed bone in the maxillofacial region that

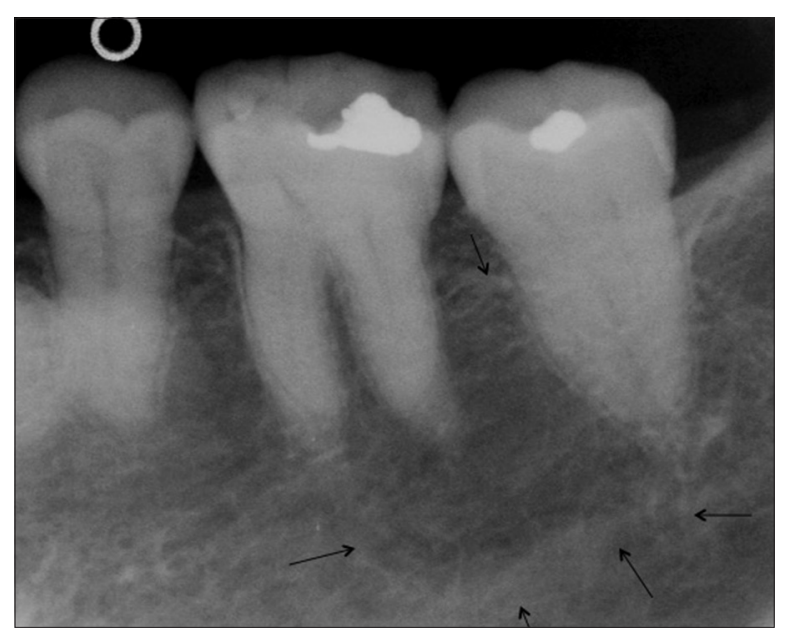

Figure 3: Clinical findings of the upper left first molar. No inflammation was seen and X-ray photography appeared normal

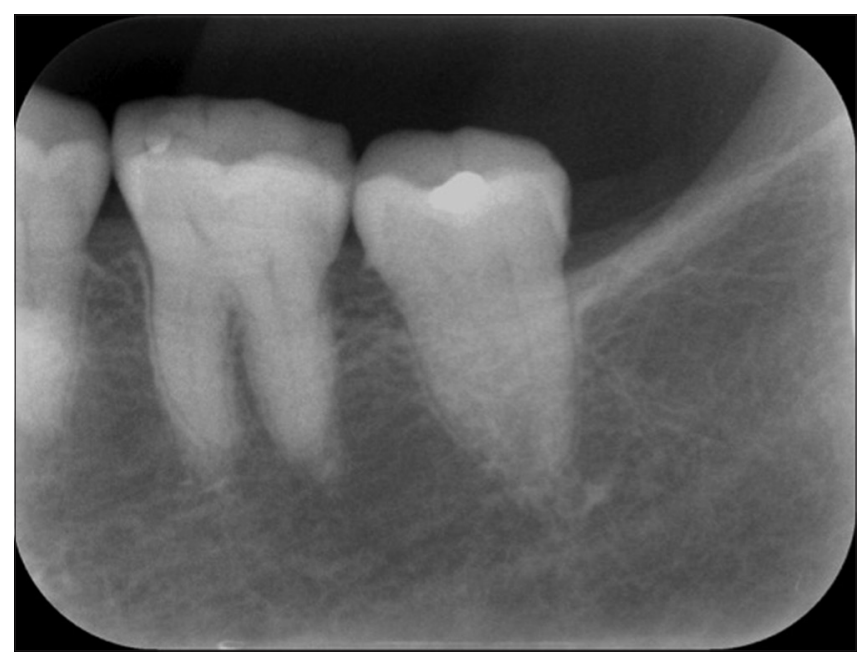

Figure 5: Radiolucent findings disappeared at the part of the lower left first and second molar

European Journal of Dentistry, Volume 11 / Issue 2 / April-June 2017 
persisted for more than 8 weeks, and (3) no history of radiation therapy to the jaw..$^{[11,12,14]}$

In 2010, the position paper on BRONJ has been reported from 5 Japanese societies (The Japanese Society for Bone and Mineral Research, Japan Osteoporosis, Japanese Society for Oral and Maxillofacial Radiology, Japanese Society of Periodontology, and Japanese Society of Oral and Maxillofacial Surgeons). ${ }^{[12]}$ In this study, BRONJ was unknown of incidence mechanism, and prevention and coping methods were not established. The cause occurring only in the jaw was as follows: (1) source of oral infection is easily invasive to jaws from the space between epithelium and tooth due to the tooth-implanted broken epithelial tissue. (2) Oral membrane was easily injured by chewing, etc., therefore, the infection invasive under the jaw covered with thin oral membrane was different from other bones. (3) In oral lesion, the source of infection was over 800 kinds, and oral bacteria were number of $10^{11}$ from $10^{12} / \mathrm{cm}^{3}$. (4) Through dental infection (caries, osteomyelitis, apical lesion, and periodontal disease), the jaws are easily infected. (5) When the jaws are directly exposed, it is easily infected through invasive dental treatment (e.g., extraction). Regarding clinical findings, differential diagnosis, risk factor, etc., have also been described. They are mostly similar previous reports..$^{[14]}$

In 2007 in Japan, the extraction for patients administrating BP medicines was an absolute contraindication. Therefore, we selected preservation therapy unavoidingly, through discussion with the oral surgeon.

Till now, occurrences of BRONJ due to denture ${ }^{[14-16]}$ and root canal treatment ${ }^{[17]}$ have been reported. However, this case of preservation method for tooth fracture was the first successful case. There are completely different opinions for BRONJ whether to perform surgical treatment or preservation treatment. Magopoulos et al. ${ }^{[5]}$ have reported that when surgical treatment was being performed at the pause medicine period under 6 months, the lesion flared. However, when the pause medicine period was over 6 months, the lesion was cured by surgical treatment. Stanton and Balasanian, ${ }^{[18]}$ Carlson and Basile, ${ }^{[6]}$ Williamson, ${ }^{[19]}$ and Engroff and $\mathrm{Kim}^{[7]}$ have also reported the lesion cured by surgical treatment. On the other hand, Migliorati et al. ${ }^{[20]}$ have reported that active surgical treatment is not effective and the enlargement of the lesion is possible to occur. Therefore, they recommend preservation method (gargle, local rinse, and long period medication of antibiotics) and minimize necessity of the removal of the necrotic tissues of the exposed bone. Ruggiero et al. ${ }^{[21]}$ have also recommended avoiding surgical treatment. At present, the pause medication period of $\mathrm{BP}$ is desirable for at least 3 months; however, we have concluded that report by Yoneda et al. ${ }^{[12]}$ and Ruggiero et al. ${ }^{[14]}$ as "the pause medication of BP affects the healing," "there is no sure evidence to lower the occurrence ratio of BRONJ even though the pause of medication is being done surgical treatment" should not be forgotten.

The most important point is to suspect the osteomyelitis of jaw caused by infection and administer antibiotics as soon as possible. Many researchers have reported that bad oral hygiene is the major risk factor. ${ }^{[10-14,22-24]}$ Therefore, we have performed complete education of BRONJ for patient and regular oral management (observation of progress every month and X-ray photographs taken every 6 months). We have concluded that these correspondences led to good progress in this case.

On the other hand, in 2009, the position paper of AAOMS has reported the Stage 0 of BRONJ. ${ }^{[14]}$ Stage 0 is as follows: patients with no clinical evidence of necrotic bone but present with nonspecific symptoms or clinical and radiographic findings, such as, symptoms: (1) odontalgia not explained by an odontogenic cause, (2) dull, aching bone pain in the body of the mandible, which may radiate to the temporomandibular joint region, and (3) sinus pain, which may be associated with inflammation and thickening of the maxillary sinus wall; clinical findings: (1) loosening of teeth not explained by chronic periodontal disease and (2) periapical/periodontal fistula that is not associated with pulpal necrosis due to caries; and radiographic findings: (1) alveolar bone loss resorption not attributable to chronic periodontal disease, (2) change to trabecular pattern - dense woven bone and persistence of unremodeled bone in extraction sockets, (3) thickening/obscuring of periodontal ligament (thickening of the lamina dura and decreased size of the periodontal ligament space), and (4) infection in alveolar canal narrowing. Furthermore, theses nonspecific findings, which characterize Stage 0, may occur in patients with a prior history of Stage 1, 2, or 3. (2009, position paper). In 2012, Shalon suggested the caution points of the radiograph of Stage 0 as follows: radiographic alterations are not evident until there is significant bone involvement. Early stage of BRONJ may not reveal significant changes on panoramic and periapical 
films. Late radiographic change may mimic classic periapical inflammatory lesion or osteomyelitis. ${ }^{[25]}$ Recently, nonexposed variant of BRONJ in oral lesion has been reported. ${ }^{[26]}$

We considered the possibility of Stage 0 for BRONJ from the symptoms found in April 2009, and through discussion with the dentist of roentgenologist, we performed the administration of antibiotics immediately. As a result, symptom disappeared. It has been reported that long-term administration of antibiotics is effective for long period. ${ }^{[27]}$ However, in our case, symptoms disappeared in a short period of time. We have concluded that early medication led to success due to consideration of the occurrence of Stage 0 of BRONJ. Hence, this case indicated the necessity of regular progress observation. Dentists should educate patients, inspect the time of course, and manage oral condition thoroughly. Furthermore, including the elderly for the risk factor of BRONJ must be considered. In the position paper of the AAOMS, elderly (over 65 years old) and others with systemic conditions (corticosteroid therapy, diabetes, smoking, drinking, bad oral hygiene, chemotherapy, periodontitis, cancer patients, etc.) were also included. ${ }^{[14]}$ With aging, systemic diseases occur easily in the elderly. We should always consider the symptoms and signs, especially when medicating BP.

From this case, we conclude that dentists should obtain enough knowledge and immediate correspondence and should impart complete education for patients undergoing Stage 0 for BRONJ.

\section{CONCLUSION}

We first reported preservation therapy for tooth fracture case upon medicating BP. Immediate responses for inflammation and symptoms and signs of Stage 0 of BRONJ led to success. We conclude that we need to perform regular and enough clinical observation and education of elderly patients having many occasion administrated BP administration.

\section{Financial support and sponsorship}

Nil.

\section{Conflicts of interest}

There are no conflicts of interest.

\section{REFERENCES}

1. Wang J, Goodger NM, Pogrel MA. Osteonecrosis of the jaws associated with cancer chemotherapy. J Oral Maxillofac Surg 2003;61:1104-7.

2. Marx RE. Pamidronate (Aredia) and zoledronate (Zometa) induced avascular necrosis of the jaws: A growing epidemic. J Oral Maxillofac Surg 2003;61:1115-7.

3. Migliorati CA. Bisphosphonates and oral cavity avascular bone necrosis. J Clin Oncol 2003;21:4253-4.

4. Migliorati CA, Schubert MM, Peterson DE, Seneda LM. Bisphosphonate-associated osteonecrosis of mandibular and maxillary bone: An emerging oral complication of supportive cancer therapy. Cancer 2005;104:83-93.

5. Magopoulos C, Karakinaris G, Telioudis Z, Vahtsevanos K, Dimitrakopoulos I, Antoniadis K, et al. Osteonecrosis of the jaws due to bisphosphonate use. A review of 60 cases and treatment proposals. Am J Otolaryngol 2007;28:158-63.

6. Carlson ER, Basile JD. The role of surgical resection in the management of bisphosphonate-related osteonecrosis of the jaws. J Oral Maxillofac Surg 2009;67 5 Suppl: 85-95.

7. Engroff SL, Kim DD. Treating bisphosphonate osteonecrosis of the jaws: Is there a role for resection and vascularized reconstruction? J Oral Maxillofac Surg 2007;65:2374-85.

8. Takahashi K, Kawabata A, Koike H, Tohnosu N. A case of osteonecrosis of the maxilla associated with the use of a bisphosphonate. Jpn J Oral Maxillofac Surg 2006;52:416. [In Japanese with English abstract].

9. Iglesias JE, Salum FG, Figueiredo MA, Cherubini K. Important aspects concerning alendronate-related osteonecrosis of the jaws: A literature review. Gerodontology 2015;32:169-78.

10. Mavrokokki T, Cheng A, Stein B, Goss A. Nature and frequency of bisphosphonate-associated osteonecrosis of the jaws in Australia. J Oral Maxillofac Surg 2007;65:415-23.

11. Urade M, Tanaka N, Furusawa K, Shimada J, Shibata T, Kirita T, et al. Nationwide survey for bisphosphonate-related osteonecrosis of the jaws in Japan. J Oral Maxillofac Surg 2011;69:e364-71.

12. Yoneda $T$, Oguno H, Sugimoto $T$, Shibata $T$, Kinta $T$, Yamamoto $T$, et al. Bisphosphonate-related osteonecrosis of the jaw: Position paper from the Allied Task Force Committee of Japanese Society for Bone and Mineral Research, Japan Osteoporosis Society of Periodontology, Japanese for Oral and Maxillofacial Radiology and Japanese Society Oral and Maxillofacial Surgeons. J Bone Miner Metab 2010;28:365-83.

13. MawardiH, Treister N, RichardsonP, Anderson K, Munshi N, Faiella RA, et al. Sinus tracts - An early sign of bisphosphonate-associated osteonecrosis of the jaws? J Oral Maxillofac Surg 2009;67:593-601.

14. Ruggiero SL, Dodson TB, Assael LA, Landesberg R, Marx RE, Mehrotra B; American Association of Oral and Maxillofacial Surgeons. American Association of Oral and Maxillofacial Surgeons position paper on bisphosphonate-related osteonecrosis of the jaws - 2009 update. J Oral Maxillofac Surg 2009;67 5 Suppl: 2-12.

15. Siegel MA, Migliorati CA, Velez I, Forrest M. Exposed bone in the palate. J Am Dent Assoc 2007;138:1341-3.

16. Migliorati CA, Armonis BN, Nicolatou-Galitis O. Oral osteonecrosis associated with the use of ibandronate: Report of a case and clinical implications. Oral Surg Oral Med Oral Pathol Oral Radiol Endod 2008;106:e18-21.

17. Sarathy AP, Bourgeois SL Jr., Goodell GG. Bisphosphonate-associated osteonecrosis of the jaws and endodontic treatment: Two case reports. J Endod 2005;31:759-63.

18. Stanton DC, Balasanian E. Outcome of surgical management of bisphosphonate-related osteonecrosis of the jaws: Review of 33 surgical cases. J Oral Maxillofac Surg 2009;67:943-50.

19. Williamson RA. Surgical management of bisphosphonate induced osteonecrosis of the jaws. Int J Oral Maxillofac Surg 2010;39:251-5.

20. Migliorati CA, Casiglia J, Epstein J, Jacobsen PL, Siegel MA, Woo SB Managing the care of patients with bisphosphonate-associated osteonecrosis: An American Academy of Oral Medicine position paper. J Am Dent Assoc 2005;136:1658-68.

21. Ruggiero SL, Mehrotra B, Rosenberg TJ, Engroff SL. Osteonecrosis of the jaws associated with the use of bisphosphonates: A review of 63 cases. J Oral Maxillofac Surg 2004;62:527-34.

22. Migliorati CA, Siegel MA, Elting LS. Bisphosphonate-associated osteonecrosis: A long-term complication of bisphosphonate treatment. Lancet Oncol 2006;7:508-14.

23. Palomo L, Bissada N, Liu J. Bisphosphonate therapy for bone loss in patients with osteoporosis and periodontal disease: Clinical perspectives and review of the literature. Quintessence Int 2006;37:103-7. 
24. Dodson TB. Intravenous bisphosphonate therapy and bisphosphonaterelated osteonecrosis of the jaws. J Oral Maxillofac Surg 2009;67 5 Suppl: 44-52.

25. Saldanha S, Shenoy VK, Eachampati P, Uppal N. Dental implications of bisphosphonate-related osteonecrosis. Gerodontology 2012;29:177-87.

26. Patel S, Choyee S, Uyanne J, Nguyen AL, Lee P, Sedghizadeh PP, et al. Non-exposed bisphosphonate-related osteonecrosis of the jaw: A critical assessment of current definition, staging, and treatment guidelines. Oral Dis 2012;18:625-32.

27. Hoefert S, Eufinger H. Relevance of a prolonged preoperative antibiotic regime in the treatment of bisphosphonate-related osteonecrosis of the jaw. J Oral Maxillofac Surg 2011;69:362-80. 